\title{
DESIGN OF A COMMAND-TRIGGERED PLASMA OPENING SWITCH FOR TERAWATT APPLICATIONS
}

\author{
M. E. Savage, C.W. Mendel, Jr.", D.B. Seidel
}

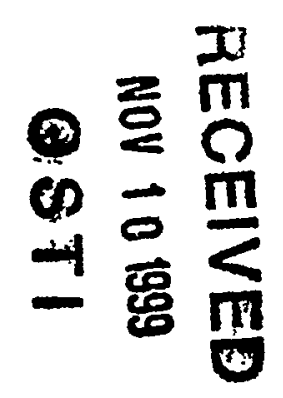

\section{ABSTRACT}

Inductive energy storage systems can have high energy density, leading to smaller, less expensive systems. The crucial element of an inductive energy storage system is the opening switch. This switch must conduct current while energy is stored in an inductor, then open quickly to transfer this energy to a load. Plasma can perform this function. The Plasma Opening Switch (POS) has been studied for more than two decades. Success with the conventional plasma opening switch has been limited.

A system designed to significantly improve the performance of vacuum opening switches is described in this paper. The gap cleared of plasma is a rough figure-of-merit for vacuum opening switches. Typical opened gaps of $3 \mathrm{~mm}$ are reported for conventional switches. The goal for the system described in this paper is more than $3 \mathrm{~cm}$. To achieve this, the command-triggered POS adds an active opening mechanism, which allows complete separation of conduction and opening. This separation is advantageous because of the widely different time scales of conduction and opening. The detrimental process of magnetic field penetration into the plasma during conduction is less important in this switch. The opening mechanism duration is much shorter than the conduction time, so penetration during opening is insignificant. Opening is accomplished with a fast magnetic field that pushes plasma out of the switch region. Plasma must be removed from the switch region to allow high voltage. This paper will describe some processes important during conduction and opening, and show calculations on the trigger requirements. The design of the switch is shown. This system is designed to demonstrate both improved performance and nanosecond output jitter at levels greater than one terawatt. An amplification mechanism is described which reduces the trigger energy. Particle-in-cell simulations of the system are also shown.

Sandia National Laboratories

PO Box 5800 Mail Stop 1194

Albuquerque, NM 87185-1194

"Cove Consulting, Albuquerque NM

\section{INTRODUCTION}

The . well-known advantages of inductive energy storage could allow smaller and more efficient pulsedpower drivers. Inductive energy storage systems allow lower voltage at the vacuum interface; the water pulsecompression elements and vacuum interface are significant cost and size items in high-power drivers. Because the opening switch is the point of highest voltage in an inductive storage design, it is the single most important part of such systems. An efficient opening switch that operates in vacuum, close to the load would have many applications. Such a switch would reduce the voltage that the vacuum insulator must withstand, and use the inductance associated with the vacuum interface to advantage as energy storage.

Since its invention, the plasma opening switch [1] has been studied as an attractive pulse-compression element for pulsed-power applications [2]. A POS exploits the fact that plasma is an excellent conductor with low mass. The mass is important because the plasma must be moved rapidly to open the switch, and lower mass conductors require less energy to move. The fact that the POS operates in vacuum also allows magnetic insulation, with higher insulation strength than any other dielectric.

Though widely studied, application of the plasma opening switch has met with limited success in demanding applications. One basic reason is the lack of a strong, abrupt mechanism for opening in the standard POS. The only force at work in the standard POS is the drive current magnetic field. This field is slow-rising compared to the desired opening time, and is almost constant near peak current (maximum stored energy). For opening, rapid removal of plasma from the switch region is necessary. An independent mechanism that quickly removes plasma from the switch region would improve POS performance. A magnetic field that rises much faster than the drive current is one way to remove that plasma.

The slow-changing drive current magnetic field also limits opening jitter of the conventional plasma opening 
switch. Since the drive current field is changing little on the opening time scale, the opening time of a conventional POS is sensitive to small changes in plasma fill density and initial conditions. This can cause jitter to be introduced by a conventional switch. Because the vacuum opening switch is the final stage of pulse compression, some applications will be intolerant of any jitter added by the POS.

The standard $\mathrm{POS}$ is realized by injecting plasma from an external plasma source into the anode-cathode gap of a magnetically insulated transmission line. Figure 1 shows schênatically simplified plasma opening switch.

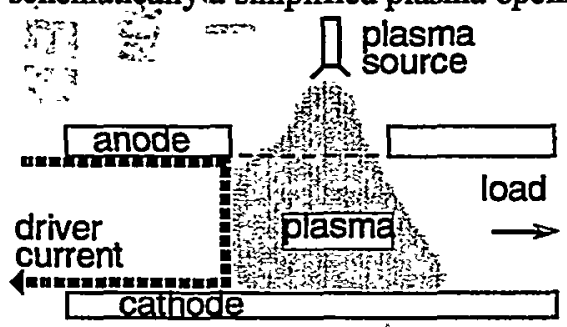

Figure 1. A simplified conventional plasma opening switch.

The plasma short-circuits the transmission line, allowing magnetic energy to accumulate in the storage inductance upstream of the plasma opening switch. When the POS opens, the stored magnetic energy is transferred to the load. There are two effects that cause the initially closed POS to open: first, there is a magnetic pressure from the storage inductor current pushing on the plasma (which has no tensile or shear strength); second, there is ion depletion from the plasma due to voltage across a sheath. At typical plasma densities and magnetic fields, ion erosion is a relatively small effect. Unless field penetration is severe (see below), it is the axial force from the storage inductor current that causes opening in a conventional POS. The switch opens, rather than simply translating a slug of plasma, largely because the plasma mass density is non-uniform across the gap. To a lesser extent, there is plasma shearing due to the variation of magnetic field with radius in the coaxial transmission lines typically used. Because the opening mechanism (storage inductor current) is applied continuously while the switch is closed, it is solely plasma inertia that keeps the conventional switch closed. This means that keeping a switch closed for longer times requires higher plasma mass. For a given conventional POS geometry and drive current, one would expect the opening time to be (at least) proportional to the conduction time.

While the plasma is conducting current, it is possible for the drive current magnetic field to penetrate into the plasma. This is one problem with conventional switches in which the opening current is the same as the (relatively slow) drive current. The penetration mechanism is replacement of plasma electrons with flux-carrying electrons emitted from the cathode conductor. Because these electrons essentially drift in the $\mathrm{E} \times \mathrm{B}$ direction, this is often called Hall penetration [3,4]. Figure 2 shows schematically the penetration of magnetic field into the plasma.

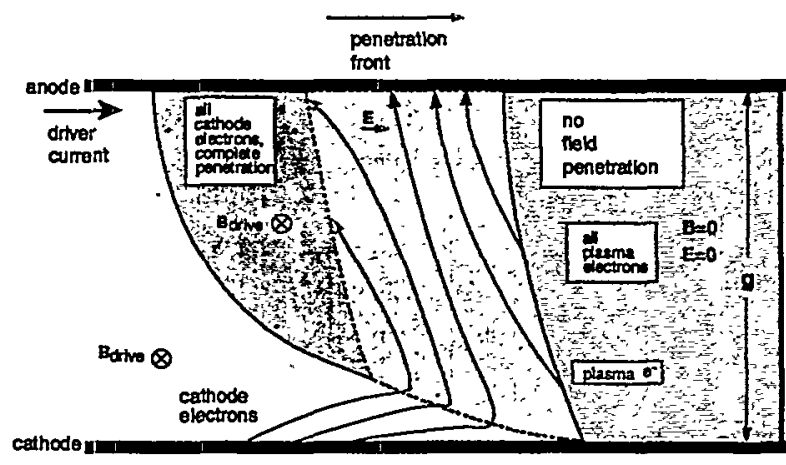

Figure 2. Magnetic field penetration into a plasma by replacement of plasma electrons with electrons emitted from the cathode.

Since the electrons in the injected plasma have zero canonical angular momentum, magnetic field cannot penetrate into that region unless the electrons are pushed away (bulk plasma translation) or replaced. The electrons emitted from the cathode can have non-zero canonical angular momentum because of momentum transfer to the cathode conductor. Magnetic field penetrates the plasma at the rate plasma electrons are replaced. Since both plasma electrons and cathode electrons carry current, the penetration rate varies with time. The rate at which original plasma electrons leave the plasma determines the penetration velocity. The velocity of replacement (penetration) is:

$$
v=\frac{I_{p e}}{2 \pi r \cdot n_{e} e \cdot g}
$$

Here $I_{p c}$ is the current in plasma electrons only, $r$ is the average switch radius, $n_{e}$ is the plasma electron density, $e$ is the electron charge, and $g$ is the mechanical gap in the switch region. Initially, plasma electrons carry the entire generator current. As the plasma electrons are replaced, cathode electrons carry more current and the penetration slows.

Assuming that the penetration rate is proportional to the number of plasma electrons remaining, one can derive an expression for the position of the magnetic field penetration front:

$$
x=x_{0}\left[1-\exp \left(\int_{0}^{t} \frac{I_{\text {gen }}}{Q_{p}} d \tau\right)\right]
$$

with

$$
Q_{p}=2 \pi r \cdot n_{e} e \cdot g \cdot x_{0}
$$

$x$ is the distance into the plasma region into which field has penetrated, $I_{g e n}$ is the generator current, and $x_{0}$ is the initial axial length of the plasma. $Q_{p}$ is the total ion charge in the plasma, which is also the charge in plasma electrons before any are replaced. The plasma ion charge changes (though usually not dramatically) as ions leave the plasma. This approximation assumes the plasma mass distribution is unchanging. Compared to particlein-cell simulation data, this simple model somewhat over-predicts the replacement rate. 


\section{DISCLAIMER}

This report was prepared as an account of work sponsored by an agency of the United States Government. Neither the United States Government nor any agency thereof, nor any of their employees, make any warranty, express or implied, or assumes any legal liability or responsibility for the accuracy, completeness, or usefuiness of any information, apparatus, product, or process disclosed, or represents that its use would not infringe privately owned rights. Reference herein to any specific commercial product, process, or service by trade name, trademark, manufacturer, or otherwise does not necessarily constitute or imply its endorsement, recommendation, or favoring by the United States Government or any agency thereof. The views and opinions of authors expressed herein do not necessarily state or reflect those of the United States Government or any agency thereof. 


\section{DISCLAIMER}

Portions of this document may be illegible in electronic image products. Images are produced from the best available original document. 
For a coaxial switch of $10 \mathrm{~cm}$ radius, $5 \mathrm{~cm}$ gap, and axial switch length of $15 \mathrm{~cm}$, and a doubly-ionized plasma at $10^{21}$ ions $/ \mathrm{m}^{3}$, the penetration time constant $\tau_{=} \frac{I_{g \text { en }}}{Q_{p}}$ is

1.5 $\mu$ s at $1 \mathrm{MA}$ drive current. Thus in such an experiment with $500 \mathrm{~ns}$ or longer, drive time, there is considerable plasma mass left behind the magnetic piston. The voltage in the switch region cannot rise appreciably until the charge density in the gap decreases to much below the initial density. These ions can only be removed by acceleration across an electric sheath, at the expense of more energy than sweeping them in a magnetic snowplow. Increasing the plasma ion number density reduces field penetration but raises the mass that must be moved for opening. A viable approach to improving opening switch performance would be to reduce the opening field rise time so that penetration is unimportant for reasonable plasma densities.

Another important vacuum opening switch parameter is the amount the switch opens. The fraction of the anodecathode gap cleared of plasma determines the efficiency of the POS. One way to infer the effective gap cleared of -plasma is to measure voltage and electron flow downstream of the POS and compute the consistent vacuum impedance. This technique is a flow impedance $[5,6]$ calculation. The flow impedance of a section of magnetically insulated transmission line is

$$
\mathrm{Z}_{\mathrm{r}}=\frac{\mathrm{V}}{\sqrt{\mathrm{I}_{u a}^{2}-\mathrm{I}_{d c}^{2}}}
$$

where $Z_{r}$ is the flow impedance, $V$ is the voltage, $I_{u z}$ is the anode current upstream of the section and $I_{\alpha}$ is the cathode current downstream of the section.

Flow impedance considers axial electron flow, which is important in magnetically insulated systems. For this reason, flow impedance may be used as a measure of opening switch performance, instead of other commonly used parameters such as voltage or resistance. For efficiency, the effective impedance of the opening switch must be higher than the load impedance, so it is the load and not the opening switch that determines voltage. Another common technique is modeling the POS as a radial resistor. This is not generally useful because the calculated resistance varies dramatically with both load impedance and current monitor location, due to axial current in vacuum-flowing electrons.

A desirable opening switch for an inductive energy store system is one that opens quickly compared to the output pulse width, and opens far enough to allow efficient energy transfer to the load. Further, since the POS is the final stage of pulse compression, a system that allows temporal synchronization of the output pulse is also desirable. The command-triggered plasma opening switch described below is designed to improve performance as well as introduce the ability to actively trigger its opening.

\section{THE COMMAND-TRIGGERED PLASMA OPENING SWITCH}

The Magnetically Controlled Plasma Opening Switch (MCPOS) relies on the fact that at typical plasma densities and magnetic fields, it is magnetic pressure that causes a POS to open. The MCPOS [7] uses an additional magnetic field created by diverting storage inductor current into a helical coil assembly located in the switch region; when so diverted this coil is in series with the storage inductor cathode. The inductance of this coil is small compared to the storage inductance, but raises the magnetic field by a factor of 3 in the switch region. This gives nearly an order of magnitude higher magnetic field pressure on the plasma, which increases the amount the switch opens. This additional field is zero until the helical field coil is energized, and then ideally rises much faster than the storage inductor current. The extension of those experiments is to drive the fast coil with an external pulser.

Figure 3 shows the concept of the command-triggered plasma opening switch. An applied magnetic field (with a peak axial field component $30-60$ percent of the peak drive current field) guides plasma into the transmission line anode-cathode gap. This applied field is supplied in a millisecond time scale, which requires a few kilovolts. This field also holds the plasma in place, countering the impulse from the drive current pressure.

While the plasma conducts the driver current, electron replacement field penetration occurs to some extent; however, the plasma density will be chosen such that the voltage drop will be less than a few kilovolts before the switch opens.

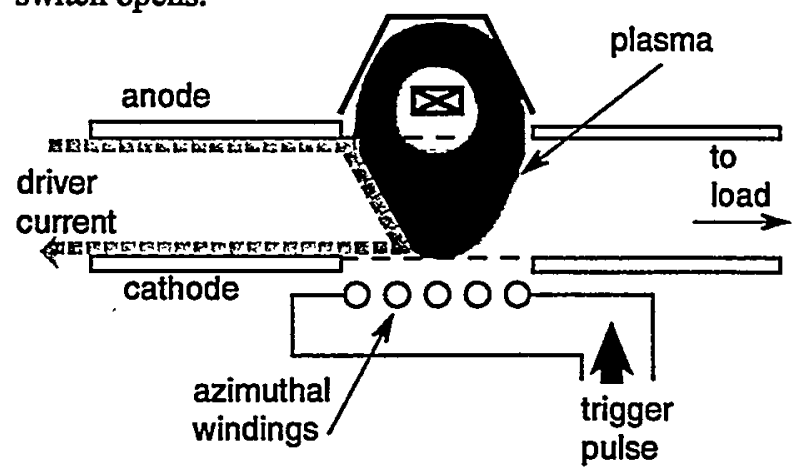

Figure 3. The triggered opening switch concept. Plasma is held in place by an applied magnetic field. The closed condition is shown.

Application of the trigger pulse energizes the azimuthal windings (the 'fast coil'). This azimuthal current causes a force on the plasma surface. This trigger field has a rise time 5-10 times faster than the storage inductor current. Whereas the storage inductor current might have penetrated substantially into the plasma, the trigger field penetration is negligible because of its relatively fast rise time. This separation of conduction and opening is the central part of this work.

Opening a POS by this method would be effective, yet it will be seen below that the trigger energy depends strongly on the amount the switch is opened. A highperformance system is one in which the POS opens relatively far and fast. For this reason, this system uses 
a stage of amplification. The fast coil drive power is less than the load power; this system has power gain. Therefore, the trigger power can be reduced with an additional stage. The system described here is one in which the triggered switch diverts storage inductor current into the fast coil of a second plasma opening switch. The gain of this system reduces the required trigger power significantly.

\section{CALCULATIONS}

A set of calculations shows the approximate energy required to trigger the switch. Because of the fast rise time of the trigger field, we will ignore field penetration.

The first requirement is that the magnetic field pressure be adequate to push the plasma. A pressure-balance calculation shows that the magnetic field must be

$$
\mathrm{B} \geq \mathrm{v}_{\text {sheath }} \sqrt{2 \mu_{0} \mathrm{n}_{0} \mathrm{~m}_{\mathrm{i}}} \text {. }
$$

Here $B$ is the magnetic field at the plasma surface, $v_{\text {sheath }}$ is the magnetic piston velocity, $\mu_{0}$ is the permeability of space, $n_{0}$ and $m_{i}$ are the plasma number density and ion mass, respectively. Note that the plasma density required stems from allowing replacement of half or less of the plasma electrons; the penetration time constant should be at least twice the conduction time. For a sheath velocity consistent with $2 \mathrm{~cm}$ in $50 \mathrm{~ns}$, the magnetic field must be about $0.5 \mathrm{~T}$.

Second, the trigger pulser must supply the energy to move the plasma and fill the volume with magnetic field. With a constant magnetic field $B_{0}$, the energy to increase the field volume is

$$
\mathrm{E}=\eta \frac{\mathrm{B}_{0}^{2}}{\mu_{0}} \mathrm{Vol},
$$

with

$$
\frac{1}{2} \leq \eta \leq 1
$$

Vol is the plasma volume. The factor $\eta$ depends upon the final energy distribution between kinetic and magnetic energy; for a fast-moving system $\eta$ will be closer to 1 . Using a constant $0.5 \mathrm{~T}$ from Eq. 5 and the 16 liter plasma volume, the maximum energy is $3.2 \mathrm{~kJ}$. This is also an upper bound on energy required because the entire plasma volume will not be displaced, and the field density is not constant over the volume. To judge the value of an additional stage, we can use Eq. 6 to compute the energy needed for the main switch. The main switch must open farther and faster; using an amplifier stage allows triggering with $3.2 \mathrm{~kJ}$ instead of the $14 \mathrm{~kJ}$ required by the main switch.

With this estimate of the driver requirements, the system design could begin. The fast field coil could be designed with a magnetostatic model because of negligible field penetration. A planar coil with 28 vanes in parallel, each making a quarter-turn gives a vacuum inductance of $32 \mathrm{nH}$. The coil is constant-pitch, with vanes everywhere having the same angle from radial. With plasma pushed completely out of the gap, achieving $0.5 \mathrm{~T}$ at the anode requires $430 \mathrm{kA}$ in this coil. The inductance under this condition is $26 \mathrm{nH}$. The field energy at $430 \mathrm{kA}$ is $2.4 \mathrm{~kJ}$ with plasma present; with no plasma the energy is $3.0 \mathrm{~kJ}$.

\section{TRIGGER DESIGN}

The trigger pulser supplies the current that energizes the fast field coil. The resulting magnetic field applies a radial force to the plasma, which causes opening. As shown before, the energy required is a few kilojoules in 50 ns. This is approximately $0.06 \mathrm{TW}$. Present technology makes this readily available. Since this is a basic experiment, the pulser design allowed for more than the minimum energy. The pulser uses available components from other experiments. A 24-stage, 100 $\mathrm{kV}$ Marx generator charges 2 parallel $7.5 \mathrm{nF}$ water capacitors. A megavolt gas switch transfers this energy into 4 parallel $7.8 \Omega$ water pulse-forming lines. The pulse-forming lines use self-closing water switches. The output of these pulse forming lines feeds through a graded vacuum interface into the fast coil. This pulser can supply more than $800 \mathrm{kA}$ within $50 \mathrm{~ns}$ into the 32 $\mathrm{nH}$ fast coil and has over $20 \mathrm{~kJ}$ available.

\section{MECHANICAL DESIGN}

Figure 4 shows the layout of the triggered POS. The storage inductor is $17 \Omega$ coaxial vacuum line (not shown) on the left side of the figure. The load is an electronbeam diode (also not shown) to the right of the figure. Typical load impedance ranges from $6-12 \Omega$. The storage inductor is $250 \mathrm{nH}$ and the peak current is $800-900 \mathrm{kA}$. The goal is to energize the main (second) POS fast coil in 10-20 ns.

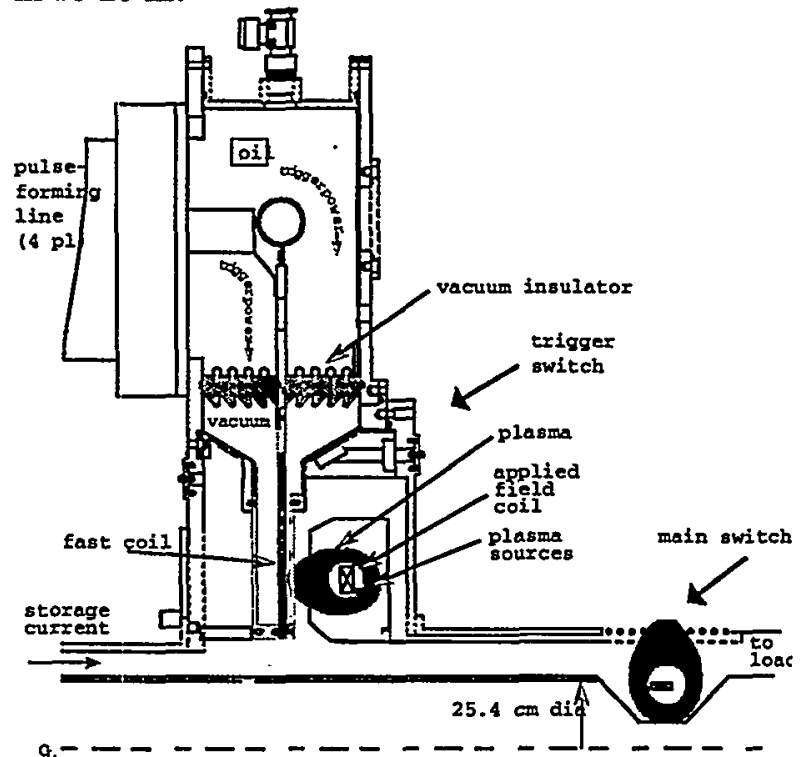

Figure 4. Cross-sectional view of the amplified-trigger POS.

\section{SIMULATIONS}

Particle-in-Cell simulations of the command-triggered POS have been run using the $2 \frac{1}{2} \mathrm{D}$ code TwoQuick [8]. A new model was added to TwoQuick that uses a tensor conductivity layer in the plane of the fast coil to properly account for the coil's current in 2D. These simulations (whose geometry, initial plasma, and slow B field are shown in Figure 5) include the double-sided fast coil feed as in the experiment, and use an electron diode load 
in place of the fast coil of the main switch. To calculate the simulations on a practical time scale, and to avoid problems due to numerical heating, the simulations are run on a timescale about one order of magnitude faster than the experiment. Scaling is done using equation 5 which says that opening rate is proportional to the square root of mass density and inversely proportional to magnetic field. The plasma mass density is about two orders of magnitude lower with $1.0 \mathrm{C} / \mathrm{m}^{3}, 1 \mathrm{AMU}$ ions (the experiment will use $32 \mathrm{C} / \mathrm{m}^{3}$ and average atomic mass of 9). The drive current is $0.5 \mathrm{MA}$, which is about half that of the experiment. The cell size in the plasma region is $0.5 \mathrm{~mm}$ and is chosen as a compromise between minimizing numerical heating and maintaining reasonable simulation cost.

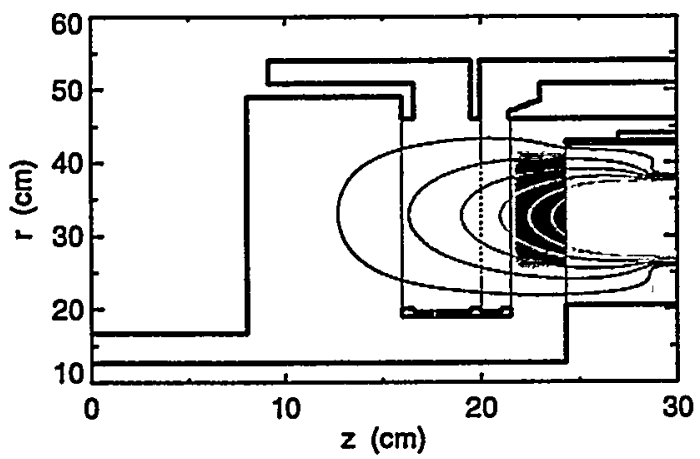

Figure 5. The simulation geometry. The fast field coil is shown and on either side of the fast coil are radial vanes (thin lines) that return the fast coil current. The applied field flux lines are shown, as is the plasma fill.

Figure 6 shows the upstream anode current, the downstream anode and cathode currents, and the fast coil (trigger) current from the simulation. For this simulation, the driver voltage is a step wave with one nanosecond risetime. The slow field is $0.2 \mathrm{~T}$ at the cathode surface. For comparison, the peak azimuthal magnetic field due to storage inductor current at this location is $0.3 \mathrm{~T}$. The trigger signal to the fast coil has a short-circuit current of $0.25 \mathrm{MA}$. It begins to rise at $1.0 \mathrm{~ns}$ after the simulation begins, but does not reach appreciable current until about $15 \mathrm{~ns}$. This time is only a factor of three faster than the experiment. The data shown in Figure 6 give a flow impedance that reaches well above $3.0 \Omega$. This is quite good considering the $4.0 \Omega$ vacuum impedance downstream of the switch.

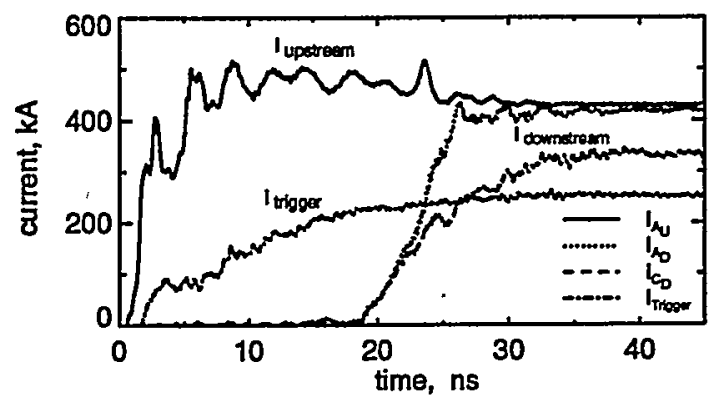

Figure 6. Simulation currents.
To test for contributions to opening from nonphysical (numerical) effects, the fast coil drive was delayed to $10 \mathrm{~ns}$, i.e. 9 ns later than above. Nothing else was changed. The delayed fast coil trigger signal resulted in a delayed switch opening (measured by flow impedance), but the rate of rise and the level of opening remained the same. If numerical heating of the plasma were an important contributor to the simulation results, significant differences would be expected in this comparison.

For efficiency and cost reasons it is desirable to minimize the trigger current supplied to the fast coil. To understand the effect of varying this current, simulations were performed at three trigger current levels: $125 \mathrm{kA}$, $250 \mathrm{kA}$, and $500 \mathrm{kA}(25 \%, 50 \%$, and $100 \%$ of drive current). In each case the slow field was adjusted in proportion to the trigger current. In each simulation, the flow impedance reaches about $3.5 \Omega$, however the 125 $\mathrm{kA}$ case takes significantly longer to open. The $500 \mathrm{kA}$ case opens slightly faster than the $250 \mathrm{kA}$ case, but not enough faster to be worth the cost. It is likely that a current lower than $250 \mathrm{kA}$ would suffice, but $125 \mathrm{kA}$ appears to be too low.

We can scale the analytic prediction of required trigger current from Section III to compare it to the simulation prediction of the required trigger level. In the full experimental conditions considered in Section III, the mass density is 32 times higher, and the timescale is 3 times longer. The full current should thus be 1.9 times larger than the simulation trigger current $(250 \mathrm{kA})$, or $475 \mathrm{kA}$. The basic analytic calculation was $430 \mathrm{kA}$ for the full system.

The simulations can readily show the relative power needed to trigger the switch and the power delivered out of the switch. Figure 7 shows the power delivered from the trigger pulser (for $250 \mathrm{kA}$ trigger current) and the power delivered out of the trigger switch.

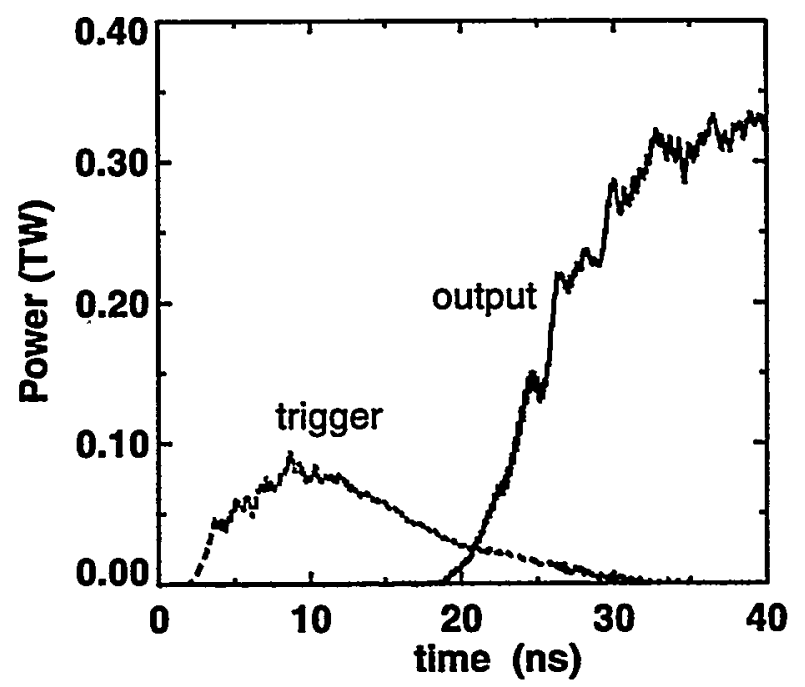

Figure 7. Simulation trigger power and power out of the trigger POS. This is from a simulation with $250 \mathrm{kA}$ trigger fast coil current. 
As discussed earlier, flow impedance is an important metric of switch performance. To demonstrate this, the $250 \mathrm{kA}$ trigger current case was repeated, varying the gap of the load diode. The gaps chosen for the three simulations performed correspond to $1.45 \Omega, 2.67 \Omega$, and $4.00 \Omega$ load impedance, respectively. Figure 8 shows the flow impedance for these three cases. The $1.45 \Omega$ load impedance data are noisy because the denominators involve a small difference between two large signals. Clearly, the figure shows that the switch flow impedance is independent of the load impedance. In contrast to the flow impedance, the switch impedance is seen to change drastically with load impedance. Clearly flow impedance is a better metric for a POS and supports the theoretical prediction that the switch flow impedance should be independent of load impedance.
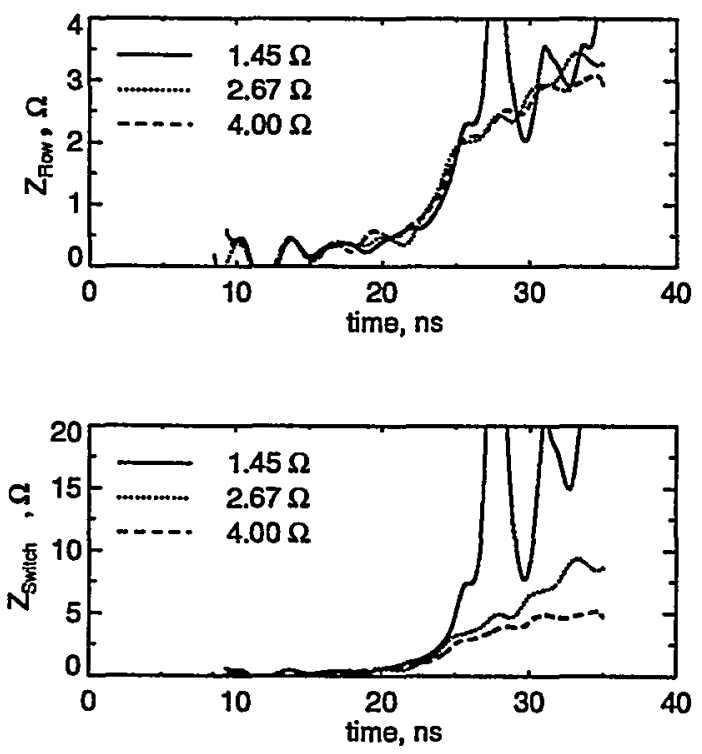

Figure 8. POS flow impedance (upper frame) and calculated resistance (lower frame) for three different load impedance values. The calculated resistance depends on load impedance, whereas flow impedance essentially does not. Note the different vertical scales.

\section{SUMMARY}

Physical considerations and computer modeling show that a command-triggered plasma opening switch is practical. This switch has advantages in its separation of conduction and opening. The independent trigger has a rise time fast enough to avoid detrimental field penetration due to electron replacement. Because each stage of this system has power gain, using a POS as an amplifier reduces the required trigger power. With this amplification, the required trigger energy will be a few $\mathrm{kJ}$ for a $100 \mathrm{~kJ}$ system.

The expected performance of the trigger stage would rapidly switch all the storage inductor current into the main switch fast coil, providing the needed large and fastrising magnetic pressure for rapid opening of that switch.

The simulations indicate that the balance in radial position and in magnetic field strengths between the fast and slow coils is important for obtaining high open flow impedance. The switch, when properly designed, appears to have excellent open flow impedance and opening speed. Simulation data show dramatically that the critical parameter of such a switch is the flow impedance, which is found to be independent of load impedance, whereas the switch resistance varies greatly depending upon the load. We have confidence in the experiment based on physical considerations and simulation results.

\section{ACKNOWLEDGEMENTS}

The authors would like to thank Dr. Dillon McDaniel for supporting this research. Work performed at Sandia National Laboratories. Sandia is a multi-program laboratory operated by Sandia Corporation, a Lockheed Martin Company for the United States Department of Energy' under Contract No. DE-AC04-94AL85000.

\section{REFERENCES}

[1] [1] C. W. Mendel, Jr. and S. A. Goldstein, "A fast-opening switch for use in REB diode experiments," J. Appl. Phys., vol. 48, pp. 1004-1006, 1977.

[2] [2] "Special issue on fast opening vacuum switches," IEEE Trans. Plasma Sci., vol. PS-15, 1987.

[3] [3] A. S. Kingsep, Y. V. Mokhov, and K. V. Chukbar, "Nonlinear skin effect in plasmas," Sov. J. Plasma Phys., vol. 10, pp. 495-499, 1984.

[4] [4] S. B. Swanekamp, J. M. Grossman, A. Fruchtman, B. V. Oliver, and P. F. Ottinger, "Particle-in-cell simulations of fast magnetic field penetration into plasma due to the Hall electric field," Phys. Plasma, vol. 3, pp. 3556-3563, 1996.

[5] [5] C. W. Mendel, Jr. and S. E. Rosenthal, "Modeling magnetically insulated devices using flow impedance," Phys. Plasmas, vol. 2, pp. 1332-1342, 1995.

[6] [6] C. W. Mendel, Jr., M. E. Savage, D. M. Zagar, W. W. Simpson, T. W. Grasser, and J. P. Quintenz, "Experiments on a current-toggled plasma-opening switch," J. Appl. Phys., vol. 71, pp. 3731-3746, 1992.

[7] [7] M. E. Savage, E. R. Hong, W. W. Simpson, and M. A. Usher, "Plasma Opening Switch Experiments at Sandia National Laboratories," presented at 10th International Conference on High Power Particle Beams, San Diego, California, 1994.

[8] [8] J. P. Quintenz, D. B. Seidel, M. L. Kiefer, T. D. Pointon, R. S. Coats, S. E. Rosenthal, T. A. Mehlhom, M. P. Desjarlais, and N. A. Krall, "Simulation Codes for Light-Ion Diode Modeling," Laser and Particle Beams, vol. 12, pp. 283-324, 1994. 